uns die traditionelle Kunstgeschichtsschreibung glauben machen will. Neben der Aufwertung weiblicher Produktionsformen, zu denen Reproduktionsarbeiten und kunsthandwerkliche Tătigkeiten gehoren, werden die Darstellungen von Frauen und Mănnern, des wWeiblichen "Mănnlichen«, hinterfragt. Die Erforschung der politischen Dimension geschlechtsgebundener Stereotypen, die in herkormmlichen Interpretationen dominieren, ist zu forcieren.

Die Re-Vision der überlieferten Deutungsmuster, Methoden und sprachlichen Formen gewinnt zunehmende Bedeutung für unser Projekt, dessen Inhalte wir in Vorträgen und Volkshochschul-Kursen zur Diskussion stellen. Aus dem großangelegten Projekt erschien uns als erster gut realisierbarer Schritt die Erstellung einer Bibliographie. Auf zwei Bănde konzipiert soll sie den Forschungsstand in der seit 1970 publizierten Literatur dokumentieren. In ihr ist der Informationsgehalt eines Handbuchs mit einem Literaturverzeichnis kombiniert, da es uns wichtig ist, auch auf Institutionen - wie Frauenarchive, -museen und -galerien aufmerksam zu machen. Bisher wurden für Band 1 ca. 6000 Titel erfaßt; dabei sind sowohl Texte mit dezidiert feministischem Ansatz als auch konventionell erarbeitete Materialsammlungen berücksichtigt worden, sofern sie für eine feministische Kunst- und Kulturgeschichte relevant sind.

Der erste Band wird wichtige Werke der Nachbardisziplinen ebenso berücksichtigen wie Titel zur feministischen, kunsthistorischen Theorie zur (historischen) Lebens-, Arbeits- und Ausbildungssituation von im Kunstbereich tătigen Frauen, zum Umgang von Frauen mit Kunst und schließlich zur Darstellung der Frau in Kunst und Kunstgeschichtsschreibung. Der zweite Band wird ausschließlich monographische Literatur zu einzelnen Künstlerinnen, Kunsthistorikerinnen, theoretikerinnen und -sammlerinnen verzeichnen. Inzwi- schen steht der erste Band der Bibliographie vor der Fertigstellung. Anfang 1990 soll die Drucklegung sein.

Für die Erstellung der Bibliographie erhalten wir seit Dezember 1986 finanzielle Unterstutzung in Form von Sach- und (zeitweise) Personalmituel durch die Universităt; zwei halbe ABM-Stellen und zusătzliche Mittel aus dem >Sonderfond für Frauenforschung des hessischen Wissenschaftsministeriums` sicherten eine Weiterarbeit an diesem Projekt bis zum März 1989. Diese Minimalfinanzierung ermoglichte uns, neben der Bibliographientatigkeit Anfragen zu Künstlerinnen, zu neuster Literatur, nach Spezialistinnen zu beantworten und BesucherInnen zu betreuen. Obwohl die finanzielle Situation seither ungesichert ist, versuchen wir die Informations- und Forschungsarbeit in beschriebener Form fortzuführen. Augenblicklich bemühen wir uns um eine Weiterfinanzierung des Projekts.
Anschrift:
FrauenKunstGeschichte,
Forschungsgruppe Marburg, c/o Kunsthistorisches Institut der Philipps-Universităt, Biegenstr. 11, 3550 Marburg, Tel.: $06421 / 282346$

\section{Der Deutsche Journalistinnenbund}

Der Journalismus gilt als Mănnerberuf. In diesem Männerberuf arbeiten ca. 10000 Frauen. Viel zu selten allerdings in leitender Position. Ihr EinfluB auf die veröffentlichte Meinung entspricht darum in keiner Weise ihrer statlichen und stăndig wachsenden Zahl. Noch immer gilt, was der Kommunikationswissenschaftler Wolfgang Langenbucher schon Mitte der 70er Jahre feststellte: 
Angesichts der offentlichen Aufgaben des Journalismus und der ihm zugeschriebenen Funktion der Meinungsbildung muß es höchst befremdlich erscheinen, daß in einem demokratischen Staat, in dem über 50 \% der Bürger Frauen sind, Informationen über weite Teile der Gesellschaft fast ausschließlich von Männern ausgewähll, beurteilt, interpretiert und vermittelt werden.

Diese Situation ist der Hintergrund der Gründung des Deutschen Journalistinnenbundes, den am 31. Oktober $198727 \mathrm{Me}-$ dienfrauen aus der Taufe hoben. Die junge Vereinigung wuchs schnell. Zur ersten Jahrestagung im Juni 1988 waren es schon 120 Mitglieder, ein Jahr spăter hatue sich die Zahl noch einmal verdoppelt. Ziel des Bundes ist es, unter den im Journalismus tătigen Frauen - unter Einschluß der Frauen in den PR- und Pressestellen - durch personliche Kontakte ein Netzwerk aufzubauen, das den Frauen in diesem angeblichen "Mănnerberuf « den Rücken stärkt: durch Kontakte, durch Informationen, durch Beratung. Zugleich möchte der Deutsche Journalistinnenbund ein Forum sein, das die Kompetenz der tătigen Frauen auch offentlich sichtbar macht: durch Tagungen, Anhorungen, Hintergrund-Gespräche. Als Sprachrohr der in ihm zusammengeschlossenen Kolleginnen wird er in kritischen Făllen auch Position beziehen. Dabei geht es gleicherweise um die Stellung der Frau in den Medien, wie um die Darstellung der Frauen durch die Medien.

Denn der Deutsche Journalistinnenbund ist mit dem Europaischen Parlament der Meinung, "daß eine verstärkte Mitwirkung der Frauen an verantwortlicher Stelle in den Medien dazu beitragen würde, den für Frauen relevanten Themen großere Aufmerksamkeit zuteil werden zu lassen, die Frauen realitătsbezogener darzustellen und zu ermutigen, sich intensiver für ihre eigenen Rechte einzusetzen “.

Der Deutsche Journalistinnenbund ist ein eingetragener Verein und als gemein- nützig anerkannt. Der Jahresbeitrag betug 1989 DM 120,-; für Berufsanfangerinnen DM 72,-. Die Teilnahme an den Jahrestagungen ist auch Kolleginnen moglich, die (noch) nicht Mitglieder im Journalistinnenbund sind. Auch die Regionaltreffen $(z \mathrm{Zt}$. in den Medienzentren Berlin, Hamburg, Düsseldorf, Koln, Frankfurt, München) sind für Găste offen.

Dem Vorstand des Deutschen Journalistinnenbundes gehoren an:

Gisela Brackert, Hessischer Rundfunk,

Frankfurt, 1. Vorsitzende

Eva Kohlrusch, Springer-Verlag, Hamburg, 2. Vorsitzende

Dr. Karin Drda-Kühn, PR \& P, Wiesbaden

Dr. Petra Lidschreiber, WDR, Köln

Gabriele Mielcke, FAZ, Frankfurt

Dr. Sybille Plogstedt, Freie, Bonn

Sabine Zurmühl, Freie, Berlin

und RA Claudia Weisbar, Frankfurt als

Schatzmeisterin.

Gisela Brackert

Adresse:

Deutscher Journalistinnenbund e.V., c/o Anwaltskanzlei Rosenbleck \& Weisbart, Grüneburgweg 3, 6000 Frankfurt 1, Tel.: 069/590931.

\section{Sabine Zurmühl}

\section{Überlegungen zum Selbstverständnis des Journalistinnenbundes}

"Der Journalist «, so bin ich vor kurzem in einem Spielfilm belehrt worden, »der Journalist muß die Wahrheit sehen und beschreiben«.

Das will ich als Journalistin auch. Die Wahrheit ist, daß es für Frauen und Mănner unterschiedliche Wirklichkeiten gibt, unterschiedliche Bereitschaft ihrer Umge- 